\title{
Alirocumab lowers atherogenic lipids in patients with metabolic syndrome to potentially lower cardiovascular risk
}

Robert R. Henry

Dirk Müller-Wieland

Pam R. Taub

Maja Bujas-Bobanovic

Michael J. Louie

Alexia Letierce

Henry N. Ginsberg

\section{Video Abstract}

Keywords: alirocumab, lipids, lipoproteins, metabolic syndrome, overweight, obese, obesity, diabetes, clinical trial, PCSK9 inhibitor, ODYSSEY, safety, efficacy, statin, cholesterol, hypercholesterolemia, LDL-C, HDL-C, VLDL-C, apolipoprotein B

Posted Date: September 20th, 2019

DOI: https://doi.org/10.21203/rs.2.15075/v1

License: (c) (1) This work is licensed under a Creative Commons Attribution 4.0 International License. Read Full License 


\section{Abstract}

Individuals with metabolic syndrome have a 2-fold increased risk of cardiovascular disease, but few strategies provide adequate cardiovascular risk reduction for this group. One option to lower this risk is to reduce atherogenic lipids, particularly low-density lipoprotein cholesterol. Statins are a recommended first-line therapy for this purpose, but this approach doesn't always provide sufficient LDL-C lowering to optimally reduce cardiovascular risk. Now, researchers have shown that alirocumab, a proprotein converstase subtilisin/kexin type 9 inhibitor, or PCSK9 inhibitor, approved for LDL-C reduction, may address this need. Pooled clinical trial data from ten phase 3 clinical trials from the ODYSSEY clinical development program showed alirocumab significantly lowered LDL-C in individuals both with and without metabolic syndrome. The analysis investigated the safety and efficacy of the drug in 4983 patients randomized to receive either treatment or control using four pools of data, grouped according to alirocumab starting dose, control, and background statin therapy. Patient baseline characteristics and safety and efficacy data were further grouped according to metabolic syndrome status. Metabolic syndrome was defined using the criteria of the International Atherosclerosis Society, but with two important differences. The range used for fasting plasma glucose was 100 to 126 milligrams per deciliter, which led to the exclusion of individuals with diabetes, and body mass index was used in place of waist circumference. The primary efficacy endpoint in all studies was the percentage change in LDL-C from baseline to week 24, analyzed using the intention-to-treat approach. Across trials, alirocumab significantly reduced LDL-C in patients with hypercholesterolemia, regardless of whether they had metabolic syndrome. Alirocumab also lowered levels of apolipoprotein B and non-HDL-C - two parameters that provide a better estimate of atherogenic particle numbers than calculated LDL-C for patients with metabolic syndrome, due to the mixed dyslipidemia that's typical of this group. Reducing non-HDL-C has also been suggested as an alternative treatment target in patients with metabolic syndrome. Importantly, alirocumab was generally well tolerated, with no apparent effect on glycemic parameters, including fasting plasma glucose and glycated hemoglobin. These results show that alirocumab has the potential to significantly reduce levels of atherogenic lipids in individuals with metabolic syndrome. By extension, the drug may also reduce cardiovascular risk in this group, providing a much-needed solution for affected individuals. 